Portland State University

PDXScholar

7-26-2016

\title{
Minimizing Corrosion of Outdoor Metalworks Using Dispersed Chemically Stabilized Nanoclays in Polyvinylidene Fluoride Latex Coatings
}

\author{
Natasja Alexandria Swartz \\ Portland State University \\ Capri A. Price \\ Portland State University \\ Tami Lasseter Clare \\ Portland State University, claret@pdx.edu
}

Follow this and additional works at: https://pdxscholar.library.pdx.edu/chem_fac

Part of the Chemistry Commons

Let us know how access to this document benefits you.

Citation Details

Swartz, N.; Price, C.; Clare, T.L. Minimizing corrosion of outdoor metalworks using dispersed chemicallystabilized nanoclays in PVDF latex coatings. ACS Omega, 2016.

This Article is brought to you for free and open access. It has been accepted for inclusion in Chemistry Faculty Publications and Presentations by an authorized administrator of PDXScholar. Please contact us if we can make this document more accessible: pdxscholar@pdx.edu. 


\title{
Minimizing Corrosion of Outdoor Metalworks Using Dispersed Chemically Stabilized Nanoclays in Polyvinylidene Fluoride Latex Coatings
}

\author{
Natasja A. Swartz, Capri A. Price, and Tami Lasseter Clare* \\ Department of Chemistry, Portland State University, P.O. Box 751, Portland, Oregon 92707, United States
}

Supporting Information

ABSTRACT: Nanoclays are small enough to appear optically transparent, yet they have large surface-to-volume and high aspect ratios that can significantly inhibit water diffusion when incorporated into protective coatings. Clear coatings, which minimally affect the aesthetics of metalworks, are commonly applied to outdoor metalworks, such as sculptures, to prevent and slow corrosion. In recent years, waterborne clear coatings, rather than solvent-based clear coatings, are increasingly used in many applications to reduce the quantity of volatile organic components in the formulation, yet the performance of dry films produced from waterborne colloidal suspensions is generally poorer. In this work, we aim to improve the barrier properties of
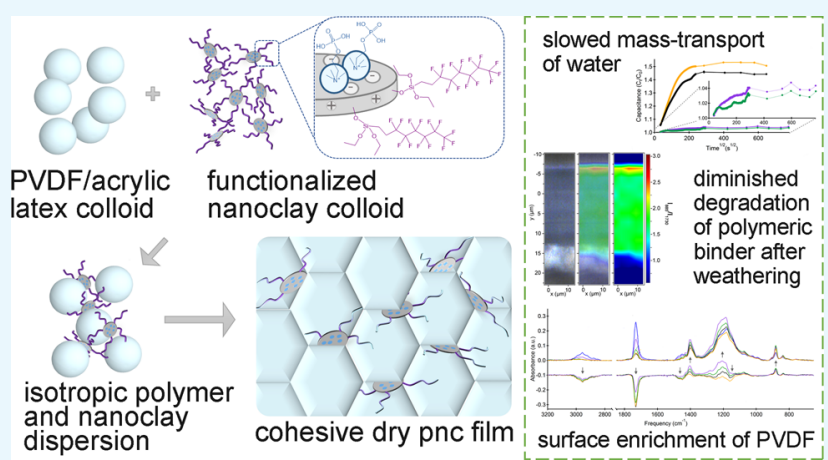
a highly weatherable waterborne acrylic/polyvinylidene fluoride and nanoclay

surface enrichment of PVDF emulsion by adding a synthetic nanoclay, Laponite, into the formulation. To improve clay-polymer compatibility, the clay was covalently modified using an acetoxy or perfluoroalkyl silane monomer that is reactive with the hydroxyl groups at the edges of the Laponite platelets. Cation exchange on the clay faces using phosphorylcholine was conducted to increase the stability in water and characterized by zeta potential. Resulting changes in barrier properties of the polymer nanocomposite films were characterized by gravimetry, colorimetry, and electrochemical impedance spectroscopy. Surface ablation after accelerated artificial weathering was monitored by attenuated total internal reflectance Fourier transform infrared microspectroscopy and Raman microspectroscopy, thin film X-ray diffraction (TF-XRD) and gloss and thickness measurements. The composite films showed many improved properties: reduced water sensitivity and ultraviolet-induced polymer degradation, which increased the barrier properties and reduced the diffusion constants over both short- and long-term weathering studies compared with films without nanoclays. The diffusion constant measured for the highest performing composite film showed that the performance gap between relevant water- and solvent-borne coatings used to protect outdoor metals was narrowed by half.

\section{INTRODUCTION}

With protective coatings, it is possible to minimize corrosion of outdoor metalworks, such as bridges, architectural elements, and sculptures, by preventing or slowing the passage of water and ions into the metal substrate. Protective coatings are often pigmented because pigment particles can act to further slow mass transport of corrosives through films. However, the use of optically transparent coatings is preferable when the ability to see the substrate is a critical feature for aesthetic and preventative maintenance reasons. Previous work by us and others has shown that polymeric coatings containing polyvinylidene fluoride (PVDF) have longer working lifetimes than polyacrylics and other polymers because of excellent chemical and thermal stability. ${ }^{1-4}$ Yet, it has also been shown that water uptake into waterborne latex coatings is greater than solvent-based coatings due to voids in the film caused by spherical close-packing defects, resulting in both a measurable decrease of barrier properties and visible water whitening of the transparent film. ${ }^{5}$ In this work, we aim to reduce mass transport into those films by incorporating optically transparent nano- clays that have been chemically modified with acetoxy or perfluoroalkylsilanes to lower their surface energies and increase their dispersibility and compatibility within the coating. Incorporation of nanoclays into the resulting dry film should create a tortuous path that would slow diffusion of ions through the coating to the metal substrate. ${ }^{6-8}$ Such composite films are expected to have increased overall barrier properties, resulting in prolonged working lifetimes and reduced corrosion of the underlying metal substrate. To our knowledge, fluorinated nanoclays have not been incorporated into waterborne PVDF/ acrylic coatings. Most previous studies involving Laponite polymer nanocomposites (PNCs) used styrene and butyl acrylate dispersions, ${ }^{9-11}$ which are not as suitable as PVDF for long-term weatherability.

Incorporation of nanosized inorganics into thermoplastic coatings should result in films having increased hydrophobicity,

Received: June 22, 2016

Accepted: July 12, 2016

Published: July 26, 2016 


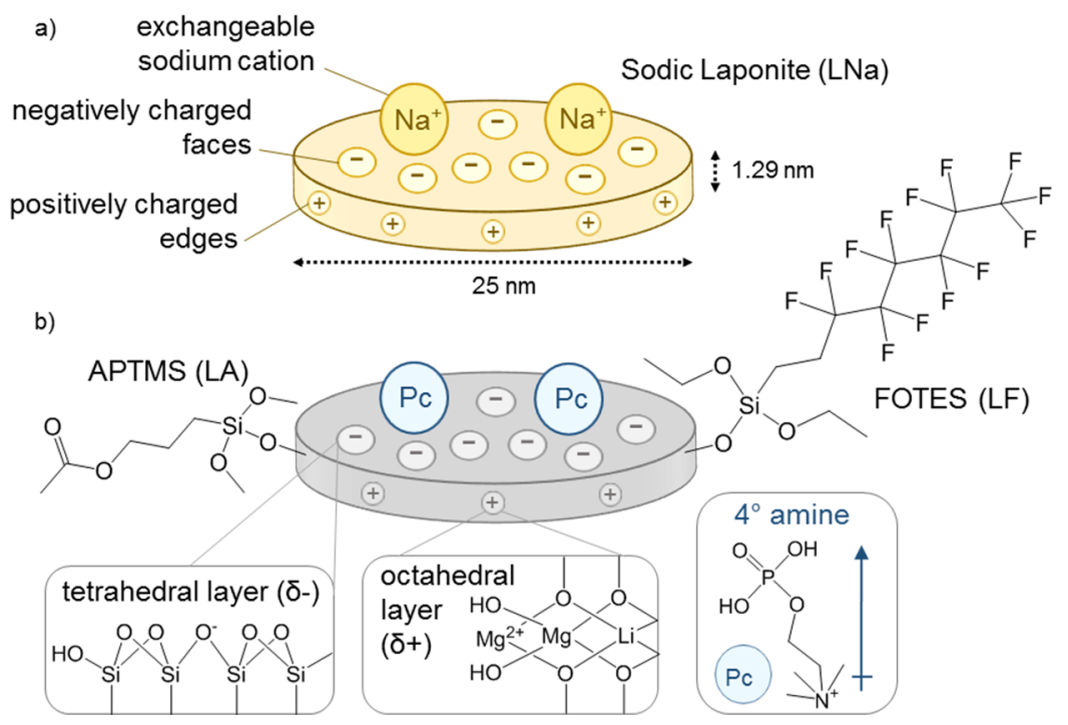

Figure 1. Single unit cell formula for Laponite is $\mathrm{Na}_{0.7}\left[\left(\mathrm{Si}_{8} \mathrm{Mg}_{5.5} \mathrm{Li}_{0.3}\right) \mathrm{O}_{20}(\mathrm{OH})_{4}\right]$. A typical $25 \times 1.29 \mathrm{~nm}$ platelet, abbreviated as $\mathrm{LNa}(\mathrm{a})$, contains approximately 1000 unit cells per discoid. Chemical modification of the clay surface (b) results in the covalent attachment of APTMS (LA) or FOTES (LF) at the tetrahedral edge and ionic exchange on the face with Pc (resulting in Laponite-APTMS-phosphoryl choline (LAPc) or LaponiteFOTES-phosphoryl choline (LFPc)).

film hardness, and inhibited water diffusion, while retaining optical clarity and easy spray application. ${ }^{12,13}$ Laponite is a synthetic silicate nanoclay in the smectite family, depicted in Figure 1A. The unique properties of clays come from their high aspect ratios (25), specific surface area $\left(350 \mathrm{~m}^{2} / \mathrm{g}\right)$, discoid single crystals, and charged surfaces. They are often used as rheology modifiers because the charged interfaces act to control diffusion in wet suspensions and as such have been used to create a range of phases from sols to gels and charged glasses. $^{14,15}$ Laponite and other smectite clays have a concentration-dependent phase diagram, where the amount, $\mathrm{pH}$, and dielectric constant of the bulk solvent play an integral role in inhibiting or facilitating particle interactions. These factors also affect the electrostatic interactions when the clay is added to a colloidal organic polymer suspension. ${ }^{16}$ The density and organization of the charges around the particle and thickness of the slipping plane can be measured by the zeta potential, where high potentials (large in absolute magnitude) lead to particle repulsion and low potentials (small in absolute magnitude) lead to flocculation due to small slipping planes that are overcome by attractive forces. At near-neutral $\mathrm{pH}$ values, expansion of sodium cations out of the interlayer space induces a negatively charged face and partial positive charge along the edge of the platelet, as depicted in Figure 1B. ${ }^{17}$ The mobile interlayer sodium cations encourage the clay to swell in water producing exfoliated (individual) 3-layered ( $\mathrm{T}-\mathrm{O}-\mathrm{T}$ ) inorganic sheets. Like many clays, Laponite is highly modifiable, in that it has both accessible hydroxyl groups available for silanation on the sheet's broken edge and exchangeable cations that balance the negative faces. ${ }^{18-21}$

The relative strength of stacking (attractive) versus dispersive (repulsive) forces must be carefully balanced to prevent nanoclay agglomeration and promote exfoliation. To produce aqueous dispersions, modification of the nanoclay was accomplished by (1) covalent reactions with acetoxy or perfluoroalkylsilanes to increase the chemical compatibility between clay and latex particles and (2) cation exchange reactions using phosphorylcholine $(\mathrm{Pc})$ to charge-stabilize the covalently functionalized nanoclays. With surface modification, the balance of those attractive/repulsive forces is altered, so the amount and type of surface coverage can have a significant influence on the dispersibility. X-ray photoelectron spectroscopy (XPS) and Raman spectroscopy were used to verify surface functionalization. Gallery spacing measurements were conducted using wide-angle X-ray diffraction (WAXD). Particleparticle interactions of wet suspensions of nanoclays and polymer were characterized by zeta potential measurements.

If not well-formulated, mechanical strain in composite films can result in cracking upon drying and/or weathering due to increased hardness and latex packing defects that increase the water volume fraction of the material. ${ }^{22,23}$ Other voids and defects in films are produced over time from the loss or surface migration of inorganic fillers (such as pigments or clays). Although there are both environmental and cost motivations to produce coatings from waterborne polymeric dispersions, the resulting films are more permeable to water due to their chemistry and film-formation mechanism. ${ }^{24}$ Adsorption of water droplets in voids that are sufficiently large to scatter white light will whiten transparent films. ${ }^{5}$ In this work, mass transport was monitored using colorimetry, gravimetry, and impedance spectroscopy.

To simulate the effects of weathering, the films were exposed to ultraviolet (UV) light and condensation cycles in an industry-standard accelerated weathering chamber (QUV). ${ }^{3}$ Electrochemical impedance spectroscopy (EIS) is a nondestructive technique for monitoring dielectric properties of materials ${ }^{5,24-26}$ and has been shown to produce similar or more sensitive results as attenuated total internal reflectance Fourier transform infrared spectroscopy (ATR-FTIR) or gravimetric methods for detecting water uptake into polymers. ${ }^{27-30}$ Films that enable facile passage of water and electrolytes (i.e., corrosives) are more poorly protective, and such films will have larger capacitances and larger diffusion constants than those that are highly protective. By fitting equivalent electrical circuits (EECs) to the EIS spectral output, the data are dissected into frequency ranges where different circuit elements are dominant. In addition, the values of each element are monitored for changes over time. Because temperature and humidity 
fluctuations during weathering eventually lead to coating failure such as cracking, time-course EIS spectra of films are used to prove insights into the electrochemical changes that occur leading up to failure. Gloss and film thickness measurements provided insights into bulk film changes.

During the lifetime of an outdoor coating, significant chemical changes may also occur that have a deleterious effect on its protective quality. The acrylic component of the PVDF resin is chemically similar to the polymer used in the primer coat of the PNCs. The acrylic polymer is a commonly used solvent-based coating for the protection of bronzes (Paraloid B44).$^{25,31-34}$ In previous work, we have observed that loss of the ester functionality is the primary means of material loss and is the pathway of the degradation for PMMA-co-PEA during outdoor and accelerated weathering. ${ }^{35}$ Although solvent-based acrylic coatings are commonly used as protective coatings, their susceptibility to chemical degradation during weathering is a notable reason to investigate more highly weatherable substitutes that offer excellent corrosion protection at a lower ultimate cost to society.

\section{EXPERIMENTAL SECTION}

2.1. Applied Coatings. Preparation of bronze and vinyl substrates is detailed elsewhere. ${ }^{5}$ Base coat primers were resindissolved in solvent [Paraloid B-44 resin, principally composed of poly(methyl methacrylate-co-ethyl acrylate), in 20\% (w/w) toluene, Dow, Inc.]. The top coat used was waterborne Kynar Aquatec FMA-12 latex [50:50, PVDF and poly(methyl methacrylate) blend, Arkema, Inc.]. Substrates (bronze and vinyl) were spray-coated with a Fuji HVLP Super 4 XPC for a dry film thickness of approximately $10-15 \mu \mathrm{m}$ for each layer (i.e., primer base coats or PVDF top coat) for a total dry film thickness of approximately $30 \mu \mathrm{m}$. Coatings were annealed in an oven for $6 \mathrm{~h}$ at $60{ }^{\circ} \mathrm{C}$. Accelerated weathering was performed according to ASTM G154 Cycle B: $4 \mathrm{~h}$ of UV-B exposure $\left(60{ }^{\circ} \mathrm{C}, E_{\mathrm{e}}=0.71\right)$ and $4 \mathrm{~h}$ of condensation $\left(50{ }^{\circ} \mathrm{C}\right.$, recirculating distilled water). For whitening and mass uptake values, films on vinyl were soaked in distilled water and analyzed at 4 and $72 \mathrm{~h}$, with three trials of each coating type. Coating masses were taken as the average of three measurements $(N=3, M=3)$, and for grayscale measurements, the films were illuminated for imaging according to ASTM D172996. Images were taken using a Nikon D40 camera and converted to grayscale in Adobe Photoshop CC, where the average $k$ value $(N=3, M=9)$ of the chart was obtained. Gloss and thickness measurements were acquired as an average of five trials using a Gardco $\mu$-Tri-Gloss meter.

2.2. Preparation of Nanoclays. Laponite $\mathrm{RD}$ and $\mathrm{S} 482$ (Southern Clay Products, Inc.) and silylating agents 3acetoxypropyltrimethoxysilane (APTMS, Gelest, Inc.) and (tridecafluoro-1,1,2,2-tetrahydrooctyl) triethoxysilane (FOTES, Gelest, Inc.) were used as supplied. To obtain covalently modified clays, toluene was distilled directly into a flask containing Laponite $\mathrm{RD}$ ( $1 \mathrm{~g}$ nanoclay/100 $\mathrm{mL}$ solvent) under anhydrous conditions in a closed system $\left(\delta^{+} \mathrm{N}_{2}\right.$ flow). After $30 \mathrm{~min}$ of heating with stirring at $35^{\circ} \mathrm{C}$, the silane was added to the toluene clay mixture ( $1 \mathrm{mmol} / \mathrm{g}$ clay), and the contents in the flask were left to stir for an additional $4 \mathrm{~h}$ at the same temperature. The covalently modified Laponite was isolated for diffraction analysis by vacuum filtration with a 0.2 $\mu$ m nylon membrane, washed extensively with toluene and then dioxane, and dried for $12 \mathrm{~h}$ at $65{ }^{\circ} \mathrm{C}$. The aqueous dispersions of the modified clay [Laponite-APTMS (LA) or Laponite-
FOTES (LF)] were obtained by quenching the grafting reaction with $50 \mathrm{~mL}$ of water and isolating the water-stable nanoclay by liquid-liquid extraction in a separatory funnel. For cation exchange, aqueous dispersions of LA and LF were diluted to $100 \mathrm{~mL}$ and heated to $50{ }^{\circ} \mathrm{C}$ after which 1 equiv $\left(\mathrm{CEC}_{\text {Laponite }}=0.75 \mathrm{mmol} / \mathrm{g}\right.$ clay $)$ of phosphocholine chloride calcium salt tetrahydrate was added and stirred for $12 \mathrm{~h}$. The exchanged/grafted clay Laponite-APTMS-phosphoryl choline (LAPc) or Laponite-FOTES-phosphoryl choline (LFPc) was vacuum filtered, washed with cold ethanol, and immediately redispersed in DI water. Aqueous dispersions $(2.6 \% \mathrm{w} / \mathrm{w})$ of clays of LAPc, LFPc, or Laponite S482 (LNa: unmodified sodic Laponite blended with peptizing agent tetrasodium pyrophosphate) were added with high shear to freshly prepared PVDF latex immediately after the preparation, and no aging/gelation of the clay was observed at the time of incorporation.

2.3. Surface Characterization. Zeta potentials were measured on filtered aqueous clay dispersions using a Malvern Zetasizer Nano and disposable plastic cells. Thermogravimetric measurements were acquired in platinum crucibles at a scanning rate of $20{ }^{\circ} \mathrm{C} / \mathrm{min}$ from 50 to $-750{ }^{\circ} \mathrm{C}$ using a PerkinElmer TGA7.

All X-ray studies were acquired using a Rigaku Ultima IV Multipurpose X-ray diffractometer with a $\mathrm{Cu} \mathrm{k} \alpha$ radiation source $(\lambda=1.542 \AA)$ and step size $=0.002^{\circ}$ for $12 \mathrm{~s}$. Powder samples were ground finely using an agate mortar and pestle and pressed into a random orientation on a mirrored slide. Thin film spectra with or without a $0.5^{\circ}$ grazing angle were acquired in situ after alignment of the sample in the $x, y$, and $z$ directions. XPS measurements were performed on vacuum dried clays using a Phi VersaProbe II with an $\mathrm{Al} \mathrm{K} \alpha$ anode (10 $\mu \mathrm{m}$ spot size) and MultiPak software.

ATR-FTIR spectra were acquired using a Nicolet Continu $\mu \mathrm{m}$ FTIR microscope with a ThermoScientific iS 10 infrared spectrometer and a $50 \mu \mathrm{m} \mathrm{MCT} \mathrm{detector}\left(4 \mathrm{~cm}^{-1}\right.$ resolution) operated with Omnic. Spectra were acquired using a diamond ATR objective to enable in situ analysis of a roughly circular area with a $250 \mu \mathrm{m}$ diameter. Data were transformed using an $\mathrm{N}-\mathrm{B}$ strong apodization function and Mertz phase correction. Raman spectra were measured with a Horiba LabRam microscope using a $532 \mathrm{~nm}$ diode laser $(22 \mathrm{~mW}), 50 \times$ objective (200 $\mu \mathrm{m}$ confocal hole), $1800 \mathrm{~g} / \mathrm{mm}$ grating, and LabSpec six software. Cross sections for maps were sliced with a scalpel, mounted on a carbon tape, and mapped in a $5 \times 15$ array with a $633 \mathrm{~nm}$ diode laser $(16 \mathrm{~mW})$.

2.4. Electrochemical Characterization. A working electrode area of $14.6 \mathrm{~cm}^{2}$ was saturated with an electrolyte $(3 \% \mathrm{NaCl})$ for EIS analysis using a Gamry Reference 600 Potentiostat from $1 \mathrm{MHz}$ to $0.1 \mathrm{~Hz}$ with AC voltage $20 \mathrm{mV}$ rms and DC voltage $0.0 \mathrm{~V}$ versus open circuit potential $(0 \pm 200$ $\mathrm{mV})$. EEC models were fit to area-normalized spectra with ZView to extract values of individual circuit elements at each time point. Errors in fit EEC models to the data were calculated as the sum of the residual error where the fit differed from the experimental data. Capacitance values $\left(C_{t}\right)$ during the immersion at time $(t)$ for diffusion analysis were calculated at $10 \mathrm{kHz}$ according to eq 1 :

$$
C_{t}=\frac{-1}{\omega Z_{\mathrm{i}}}
$$

where $\omega$ is the radial frequency and $Z_{\mathrm{i}}$ is the imaginary component of the impedance. The rate of diffusion (D) through the coatings by the most mobile species (water) was 
determined by fitting the linear portion of the relative uptake profiles to eq 2 :

$$
\frac{\log \left(C_{t} / C_{0}\right)}{\log \left(C_{s} / C_{0}\right)}=\left(\frac{4 D}{L^{2} \pi}\right)^{1 / 2} t^{1 / 2}
$$

where $C_{0}$ is the capacitance of the film before the electrolyte immersion found by extrapolation to $t=0 \mathrm{~s}, C_{\mathrm{s}}$ is the saturated capacitance of the film, $L$ is the thickness of the coating, and $t^{1 / 2}$ is the square root of the immersion time.

\section{RESULTS AND DISCUSSION}

\subsection{Effects of Surface Modification on Nanoclay} Stability. Covalent modification of Laponite with APTMS or FOTES silanes occurs via a reaction between the hydroxyl groups on the edge of the clay sheet and a silane bond to produce covalent siloxane bonds, as illustrated by the products shown in Figure 1B. Initial grafting reactions produced organophilic nanoclays that were not stable in water. To promote the exfoliated state (aqueous isotropic), ionic exchange with Pc tetrahydrate was carried out to create covalently modified and cation-exchanged nanoclays, abbreviated as LAPc and LFPc. In addition to surface coverage and grafting efficiency, the presence of chemical modifiers at each step of modification was initially confirmed by ATR-FTIR ${ }^{35}$ and then investigated by XPS, WAXD, TGA, and Raman spectroscopy; a summary of these data is presented in Table S1 and sections $1-3$ of the Supporting Information (SI).

Having hydrophilic nanoclays is important to prevent flocculation or gelation upon addition to the aqueous latex coating dispersion. Both the polar tail of Pc and the occasional exchange of monovalent sodium for a divalent calcium counterion can increase the hydration sphere and water sorption of the nanoclay particles. Characteristic Raman peaks of the layered silicates are shown in Figure 2 and include a number of $\mathrm{O}-\mathrm{H}$ stretches from metal-hydroxyl complexes as well as water adsorbed in various conformations: 3710-15 $\mathrm{cm}^{-1}(\mathrm{Mg} / \mathrm{Li}-\mathrm{OH}), 3688 \mathrm{~cm}^{-1}(\mathrm{Si}-\mathrm{OH}), 3620 \mathrm{~cm}^{-1}$ (inner sheet $\mathrm{M}-\mathrm{OH}$ ), $3425 \mathrm{~cm}^{-1}$ (amorphous $\mathrm{H}-\mathrm{OH}$, bulk water), and $3200 \mathrm{~cm}^{-1}$ (in-phase $\mathrm{H}-\mathrm{OH}$ with crystalline structure).

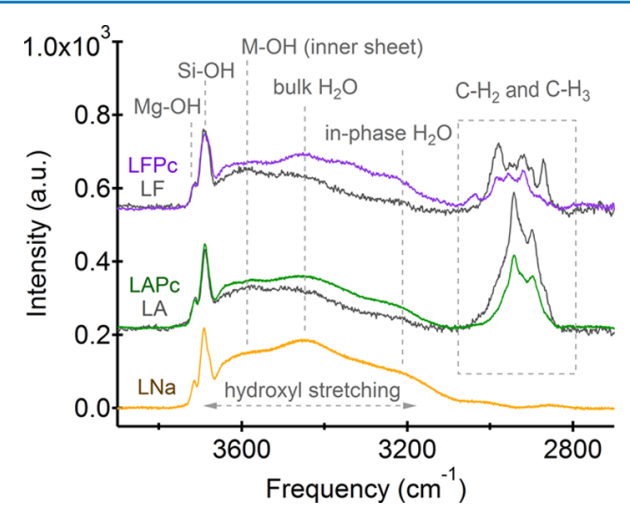

Figure 2. Raman spectra of the hydroxyl and aliphatic regions of unmodified and functionalized nanoclays, normalized to the $\mathrm{Si}-\mathrm{O}_{4}$ band at $683 \mathrm{~cm}^{-1}$. There are typically 1.4 water molecules coordinated to each $\mathrm{Na}^{+}$and 4.4 water molecules coordinated to each $\mathrm{Ca}^{2+}$ (the counterion for $\mathrm{Pc}$ used in the cation exchange). There are two hydroxyl binding sites in the clay crystal structure, $\mathrm{M}-\mathrm{OH}$ denotes inner sheet hydroxyls attached to a silicon $(\mathrm{Si}-\mathrm{OH})$ or magnesium $(\mathrm{Mg}-\mathrm{OH})$. The vibrations of external hydroxyls are at higher frequencies as labeled.
Hydrophilicity was determined by the intensity ratio of the Raman shift of bulk water (at $3450 \mathrm{~cm}^{-1}$ ) to the silicon oxide lattice of the nanoclay $\left(\mathrm{Si}-\mathrm{O}_{4}\right.$ at $683 \mathrm{~cm}^{-1}$, shown in Figure S1). ${ }^{36}$ The hydroxyl stretching bands shown in Figure 2 indicate that the largest hydrophilicity ratio was observed for the unmodified clay $\left(I_{\mathrm{H}_{2} \mathrm{O} / \mathrm{Si}-\mathrm{O}_{4}}=0.37\right)$. Bulk water was reduced significantly upon modification with organosilane and subsequently increased after the cation exchange ( $\mathrm{LA}=0.18$ to $\mathrm{LAPc}=0.29$ and $\mathrm{LF}=0.12$ to LFPc $=0.19$ ). Similar trends were observed by TGA where the unmodified clay LNa had twice as much total water adsorbed by mass than that of LA or LF (Figure S3). The higher water content in the Pc-exchanged clays is consistent with having successfully transitioned the covalently modified nanoclays from organophilic to hydrophilic after the cation exchange.

Clays are considered to be stable (i.e., unlikely to flocculate) in dilute dispersions that have zeta potentials, $\zeta$, more negative than $-30 \mathrm{mV} .{ }^{37}$ Unmodified $\mathrm{LNa}$ had a $\zeta=-42.9 \mathrm{mV}$ when dispersed in water at neutral $\mathrm{pH}$. The grafted/exchanged clays had slightly increased potentials of approximately $-38 \mathrm{mV}$ for both LAPc and LFPc. We have previously used small-angle Xray scattering (SAXS) when formulating the latex-clay suspensions to ensure adequate exfoliation in both aqueous solutions and the polymer latex suspension. ${ }^{35}$ In that work, it was noted that cation-exchanged clays (LAPc and LFPc) were stable in water, whereas those that had not been exchanged (LA and LF) were not stable in water without the addition of a peptizing agent. From these combined data, we demonstrate that such modifications to clays can result in aqueous stable dispersions.

3.2. Barrier Properties of Waterborne PVDF-Clay Nanocomposites. 3.2.1. Mass Transport of Fresh and Annealed Coatings. The grayscale values of fresh and annealed PNC films on a black substrate measured after water-soaking for 4 and 72 hours are shown in Figure 3, where black $=0 \%$ for

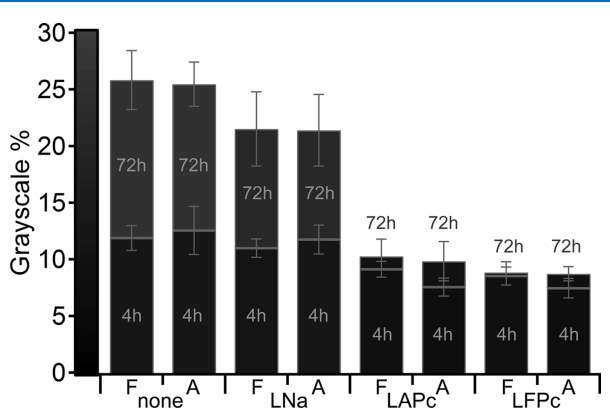

Figure 3. Whitening of the fresh (F) or annealed (A) films on black reference substrates after soaking in the electrolyte for 4 and $72 \mathrm{~h}$. The initial grayscale values of all films were approximately $8 \%$.

a transparent film and white $=100 \%$ for a completely opaque film. After soaking for $4 \mathrm{~h}$, the values were similar across films. However, sustained soaking for $72 \mathrm{~h}$ showed that water whitening was decreased by $50-65 \%$ for films with modified nanoclays compared with those without soaking. These data suggest that the numbers and/or volumes of light-scattering voids present in the final films were reduced by adding modified nanoclays LAPc and LFPc into the wet suspensions but not unmodified LNa.

Permeability tests of the fresh (dry but not weathered) films were conducted by allowing each coated substrate to soak in the electrolyte until saturation, and the capacitance was 
(a)

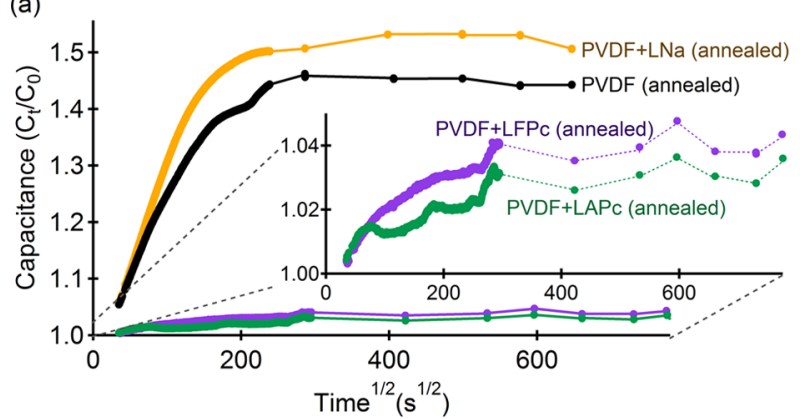

(b)

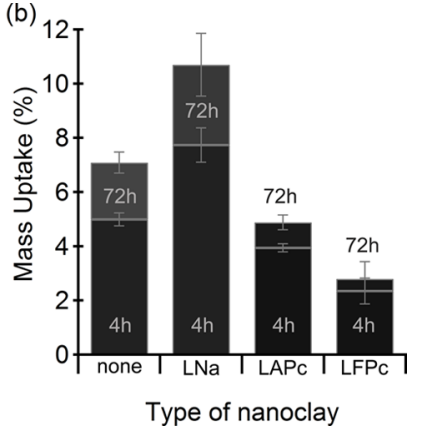

Figure 4. (a) Diffusion profiles of the electrolyte using a relative capacitance of the films over time, with the inset provided for visual clarity of the two films. (b) Mass uptake percentage of various annealed coatings after soaking in water for 4 and $72 \mathrm{~h}$. The shade of each bar represents the grayscale value shown in Figure 3.

Table 1. Barrier Properties of the Films before and after $4500 \mathrm{~h}$ QUV-B ${ }^{a}$

\begin{tabular}{|c|c|c|c|c|c|}
\hline & $D_{\mathrm{H}_{2} \mathrm{O}}\left(\mathrm{cm}^{2} / \mathrm{s}\right)$ fresh & $D_{\mathrm{H}_{2} \mathrm{O}}\left(\mathrm{cm}^{2} / \mathrm{s}\right)$ annealed & $|Z|_{0.1 \mathrm{~Hz}}\left(\mathrm{M} \Omega \cdot \mathrm{cm}^{2}\right)$ initial & $|Z|_{0.1 \mathrm{~Hz}}\left(\mathrm{M} \Omega \cdot \mathrm{cm}^{2}\right)$ QUV $4.5 \mathrm{k} \mathrm{h}$ & electrochemical stress index \\
\hline PVDF & $2.4 \times 10^{-9}$ & $4.8 \times 10^{-10}$ & 536 & 34.8 & 2.66 \\
\hline $\mathrm{PVDF}+\mathrm{LNa}$ & $5.0 \times 10^{-9}$ & $5.3 \times 10^{-10}$ & 2910 & 417 & 1.48 \\
\hline $\mathrm{PVDF}+\mathrm{LAPc}$ & $8.6 \times 10^{-10}$ & $3.7 \times 10^{-10}$ & 3490 & 1530 & 0.993 \\
\hline PVDF + LFPc & $7.6 \times 10^{-10}$ & $2.6 \times 10^{-10}$ & 4210 & 2390 & 0.987 \\
\hline
\end{tabular}

${ }^{a}$ For comparison, diffusion constants $\left(D_{\mathrm{H}_{2} \mathrm{O}}\right)$ for other types of annealed coatings: wax $=1.31 \times 10^{-9} \mathrm{~cm}^{2} / \mathrm{s}$, solvent-based acrylic $=1.46 \times 10^{-10}$ $\mathrm{cm}^{2} / \mathrm{s}$, and waterborne PVDF 70:30 $=3.95 \times 10^{-10} \mathrm{~cm}^{2} / \mathrm{s}$.

monitored as an impedance measurement at $10 \mathrm{kHz}$, using the imaginary part of the impedance as the capacitance. Relative capacitance $\left(C / C_{0}\right)$ of annealed films versus $\sqrt{t}$ is plotted in Figure 4. The traces showed that diffusion through the films occurred in two stages: initial linear uptake and then a slow percolation until the films became saturated. The initial uptake profiles $\left(35-200 \mathrm{~s}^{1 / 2}\right)$ of annealed PVDF and PVDF + LNa are considered to be type II Fickian diffusion, meaning that the pores are large enough that they do not restrict the movement of ions. In contrast, the decreased slopes of the films with LFPc and LAPc are indicative of a material having a more constrained diffusion profile than for the other two films. The diffusivity of each coating to water can be quantified by the time it takes to saturate a film and the magnitude of the films' capacitance. ${ }^{26-29}$ The diffusion coefficients of water $\left(D_{\mathrm{H}_{2} \mathrm{O}}\right)$ through the fresh and annealed films are listed in Table 1. The fresh PVDF coating with the unmodified clay $\mathrm{LNa}$ had the freest ion transport, $5 \times 10^{-9} \mathrm{~cm}^{2} / \mathrm{s}$, and developed visible pores on the coating surface upon extended soaking times. Fresh coatings with LAPc and LFPc had diffusion rates that were approximately 6 times slower than for fresh LNa- and PVDFonly films. In annealed films, the incorporation of LFPc into the PVDF coating halved its diffusion coefficient. The relatively free diffusion observed in the LNa composite film compared to the film without clay suggests that the high surface energy of the clay resulted in aggregation in voids at the surface that are large enough to scatter light, ${ }^{38}$ decreasing the film whitening (as voids would be filled with the clay) and increasing the mass uptake (as the unmodified clays are highly charged). For comparison, diffusion through the best performing film (LFPc) was larger than that of the industry-standard solvent-borne acrylic coating by only a factor of 1.8 . Calculations have demonstrated that ion movement is restricted when pores are narrowed to less than twice the diameter of the diffusing species. $^{26,39,40}$ For water, having a diameter of $\sim 0.3 \mathrm{~nm}$, the size when movement becomes restricted is approximately $0.6 \mathrm{~nm}$; therefore, the data suggest that any pores that exist in the LAPc and LFPc films are approximately $0.6 \mathrm{~nm}$ or smaller.

Having gained an understanding of the presence of lightscattering voids and permeability profiles of the composite films, the entire water content within the soaked films was measured by mass uptake studies. The data shown in Figure $4 \mathrm{~b}$ reveal that there is a much larger mass increase for the annealed films of PVDF and PVDF + LNa than for those with modified nanoclays after soaking for both 4 and $72 \mathrm{~h}$. Aside from having higher surface energy and clay-filled voids, LNa films absorbed significantly more water than the other nanocomposite films may be because of a nonreversible expansion of the mineral structure of LNa upon heating (annealing) or soaking, ${ }^{41}$ which is a response that should be reduced after the cation exchange (i.e., as in LAPc and LFPc). It is also possible that unmodified nanoclays are more easily washed out during soaking, resulting in the production of voids formerly occupied by the clays.

It is likely that films containing modified nanoclays showed improved barrier properties for two reasons. First, the nanoclay itself provided a tortuous pathway for water. Second, the covalently linked molecules have similar chemistry as the polymer (with APTMS being similar to the acrylic portion and FOTES being similar to the PVDF portion), reducing the surface energy difference between the polymer and clay and encouraging the formation of an interpenetrating network in the composite, rather than clay aggregation in the interstitial voids. From the very small increase in the whiteness value observed after $72 \mathrm{~h}$ compared with $4 \mathrm{~h}$ soaking times of the modified composite films, the data also suggest that annealing after application is not necessary for those films contrary to previous results for this latex binder. ${ }^{5}$ Although a significant reduction in mass transport was shown in modified composite films, remaining questions include differentiating the effectiveness of the acrylic versus the perfluoroalkyl-modified clays in corrosion prevention and evaluating the long-term performance profiles of the composite films. 
3.2.2. Impedance and Circuit Values as Metrics of Protective Quality during Weathering. To address these questions, weathering of the films with characterization by EIS was carried out to gain greater insight into the transport of electrolytes through the films over time. Aggressive weathering conditions were used to determine if either chemicalmodification scheme provided a significant improvement in long-term performance.

Total impedance at low frequencies $\left(|Z|_{0.1 \mathrm{~Hz}}\right)$ is usually dominated by the coating resistance $\left(R_{\text {coat }}\right)$ or charge transfer $\left(R_{\mathrm{ct}}\right)$ resistance when the $|Z|$ is large. Decreases in the value of the impedance at $0.1 \mathrm{~Hz}$ are correlated with decreases in film barrier properties, where $10 \mathrm{M} \Omega$ is the minimum acceptable value of protective films ${ }^{42}$ (or in our cell setup, $146 \mathrm{M} \Omega \cdot \mathrm{cm}^{2}$ ). Impedance values for the nanocomposite and control films are listed in Table 1 and plotted for more than $4500 \mathrm{~h}$ of weathering in Figure 5. Low-frequency impedance increased for
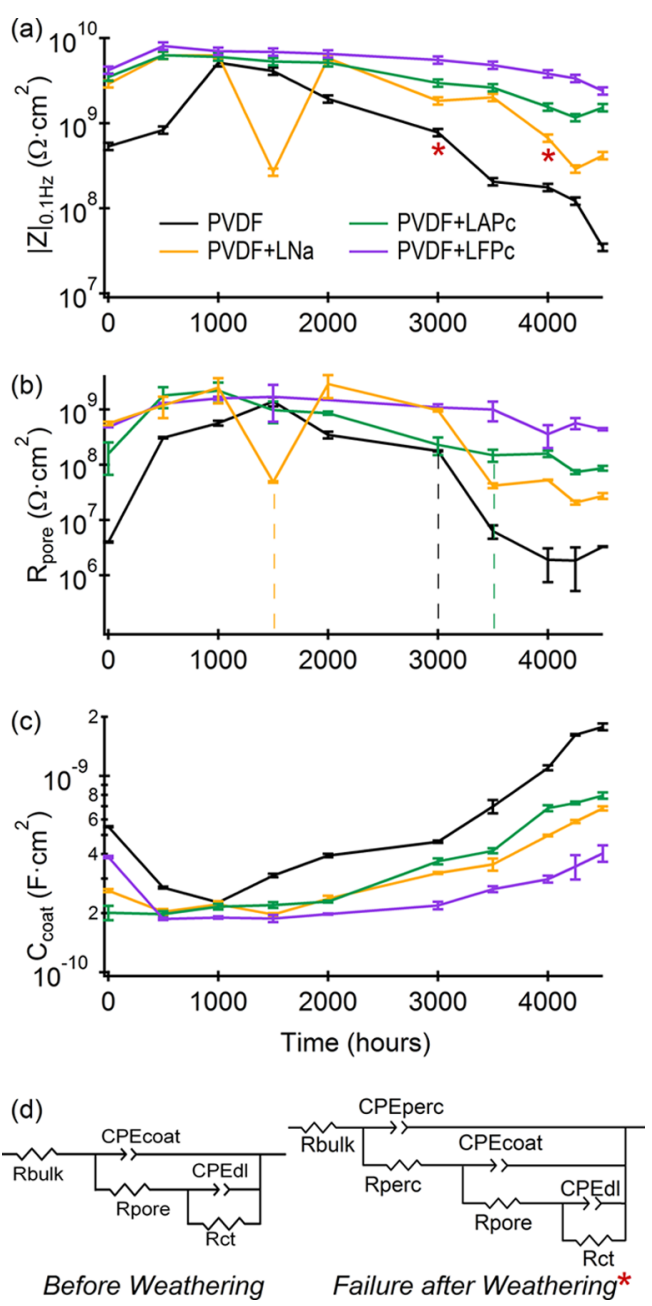

Figure 5. Impedance analysis of the films with and without nanoclays. Plots of (a) total impedance at low frequency $0.1 \mathrm{~Hz}$ as measured for more than $4500 \mathrm{~h}$ of QUV-B weathering, where the asterisk $(*)$ marks the transition to a more complex EEC, when percolation through the top layer of the coating became measurable. Coating resistance (b) and capacitance (c) are plotted using the "Before Weathering" model in (d) until $3000 \mathrm{~h}$ for PVDF and $4000 \mathrm{~h}$ for PVDF + LNa; thereafter, the "Failure after Weathering" model in (d) was used. Dashed vertical lines in (b) mark the time point when corrosion on the substrate became visible as small circular brownish dots on the samples. all films upon QUV weathering, likely because of annealing and other film morphological/chemical changes, before it decreased from its maximum value after $1500 \mathrm{~h}$ of exposure. By this metric, the LFPc film was the best protective coating as it retained a high initial impedance of $4210 \mathrm{M} \Omega \cdot \mathrm{cm}^{2}$ at $0.1 \mathrm{~Hz}$ during weathering with a final value of $2390 \mathrm{M} \Omega \cdot \mathrm{cm}^{2}$ after weathering $(-43 \%)$. The nanoclay-free PVDF control film was the least protective with an initial impedance of $536 \mathrm{M} \Omega \cdot \mathrm{cm}^{2}$ and the most drastic decrease to $34.8 \mathrm{M} \Omega \cdot \mathrm{cm}^{2}$ (-93\%), below the minimum acceptable value. The film with added LNa had an initial impedance of $2910 \mathrm{M} \Omega \cdot \mathrm{cm}^{2}$, which showed improvement over PVDF without the nanoclay and retained overall barrier properties during weathering. Although a final impedance of $417 \mathrm{M} \Omega \cdot \mathrm{cm}^{2}$, a decrease of $-86 \%$ approached the minimum protective impedance value for coatings. The coating with LAPc showed a similar performance to LFPc films with a high initial impedance of $3490 \mathrm{M} \Omega \cdot \mathrm{cm}^{2}$ and a relatively small $(-53 \%)$ decrease in barrier properties yielding a final impedance of $1530 \mathrm{M} \Omega \cdot \mathrm{cm}^{2}$. As predicted by the small mass transport values, this EIS data shows that coatings with chemically modified nanoclays retained their protective qualities the most during weathering.

It is also useful to consider changes in key EEC elements compared with their initial values over time. From model-fit values of the pore resistance and coating capacitance in Figure $5 b, c$, a similar trend to impedance values is observed and is discussed in more detail in section 4 of the SI. The polymeronly film showed the smallest relative pore resistance and largest relative coating capacitance, whereas the polymer with added LFPc showed the largest relative pore resistance and smallest relative coating capacitance. With the exception of the LFPc film, small spots of corrosion appeared on the other coated substrates at the indicated time points (dashed lines) in Figure 5b. Notably, the LNa film showed corrosion at the earliest time point $(1500 \mathrm{~h})$, possibly because of the loss of the clay from that film. In addition, each increase in the impedance of the LNa film (2000 and $3500 \mathrm{~h}$ ) was accompanied by an increase in $R_{\mathrm{c} v}$, which indicated that growth of a passivating layer of corrosion was filling in pores. Similar observations were made for LAPc at a much longer exposure time of $4500 \mathrm{~h}$. Small layers of corrosion at the bottom of pore channels offered limited protection, as their resistance was eventually overcome during soaking studies. By all of these EIS measures, LFPc was the only coating that did not develop visible corrosion and had a significantly better performance than the other films during accelerated weathering.

Another measure of a film's ability to prevent corrosion is the time constant $\left(T_{\text {coat }}=C_{\text {coat }} R_{\text {pore }}\right)$, which is discussed for each film in section 4.1 .3 of the SI. Both initially and throughout weathering, the resin-only control film performed the poorest with respect to each circuit element and had the lowest overall barrier properties. Addition of any clay increased the time constant to 5-45 times that of the polymer-only coating.

3.2.3. Electrochemical Stress Index Changes after Weathering. Using maximum and minimum values from the timecourse EIS spectra of key elements in the EEC in Figure 5d, mechanical stress within a film can be estimated by an electrochemical stress (ES) index ${ }^{43}$ shown in eq 3 : 


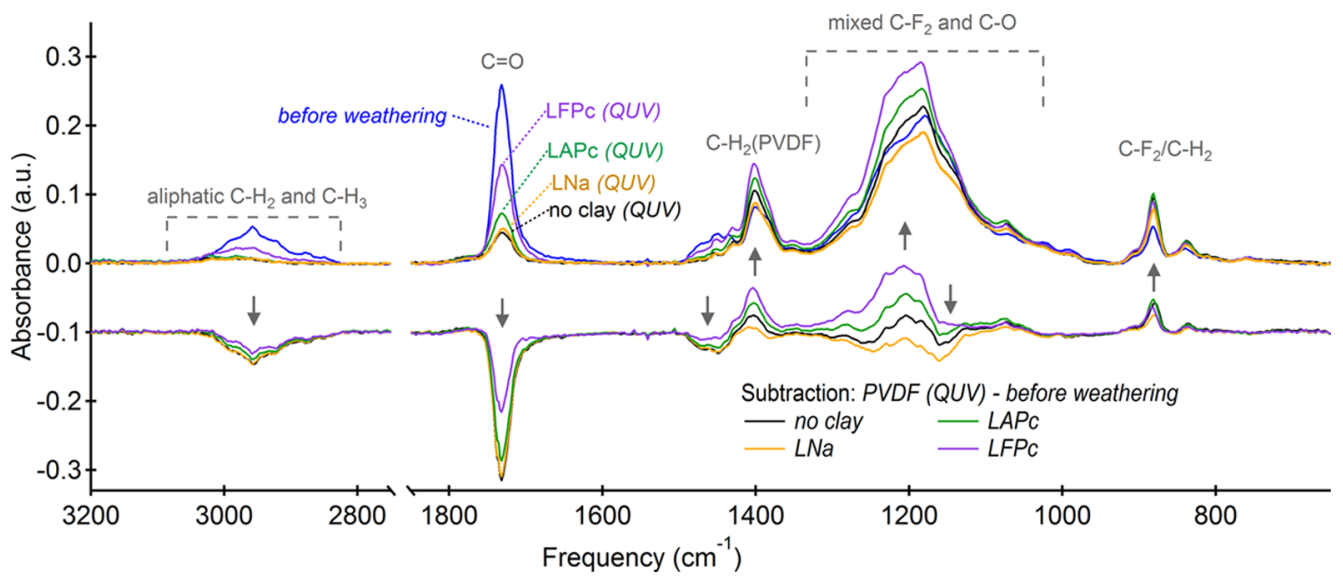

Figure 6. FTIR-ATR spectra of coated bronze panels before and after $4000 \mathrm{~h}$ of weathering by QUV. The subtraction results were produced from the weathered minus the initial (before weathering) spectrum and are vertically offset by -0.1 a.u. for visual clarity.

$$
\begin{aligned}
\mathrm{ES}= & \left(\log \left[R_{\text {pore }(\max )} / R_{\text {pore }(\min )}\right]+\log \left[R_{\mathrm{ct}(\max )} / R_{\mathrm{ct}(\min )}\right]\right. \\
& \left.+\log \left[C_{\text {coat }(\max )} / C_{\text {coat }(\min )}\right]+\log \left[C_{\mathrm{dl}(\max )} / C_{\mathrm{dl}(\min )}\right]\right) \\
& /\left(\log 1 \times 10^{5}\right)
\end{aligned}
$$

In this calculation, a resulting value close to zero indicates low stress, whereas a value of 3 is the theoretical maximum. By this metric, the PVDF resin (control film without the nanoclay) had an $\mathrm{ES}=2.66$ after $4500 \mathrm{~h}$ of weathering, which was the highest stress index in the study and makes this coating the least weatherable. Incorporation of the nanoclay had positive effects on reducing the stress index of the material: the unmodified clay $\mathrm{LNa}$ had an $\mathrm{ES}=1.48$ and films with modified clays showed even smaller changes where ES $=0.993$ for LAPc and $\mathrm{ES}=0.987$ for LFPc. From these data, incorporation of nanoclays reduces the stress index of this PVDF coating, while the magnitude of that change was dependent on the chemical modification of the nanoclay.

3.3. Photochemical Changes of Nanoclay Composites during Weathering. The data presented thus far showed that addition of chemically modified nanoclays significantly improves the short- and long-term barrier properties of an already highly weatherable coating. In an ATR-FTIR study, we observed that acrylic side groups from the polymer were lost with weathering, as shown by the subtraction spectra of PVDF/ acrylic films in Figure 6, and that the losses were not equal across all films. It is useful to note that many of the degradation products of acrylic polymers are volatile and thus are not detected, especially near the surface. In the IR subtraction spectra, each negative peak is associated with the acrylic portion of the resin, whereas nearly all positive peaks are attributed to the PVDF portion. Specifically, loss of acrylic groups is readily observed by a large negative peak at $1731 \mathrm{~cm}^{-1}$ associated with the carbonyl in the acrylate (a functional group not present in PVDF). Additional loss of aliphatic methyl character was observed at $2989 \mathrm{~cm}^{-1}\left(\mathrm{CH}_{3} \nu_{\mathrm{as}}\right), 2877 \mathrm{~cm}^{-1}\left(\mathrm{CH}_{3} \nu_{\mathrm{s}}\right)$, and $1472 / 1435 \mathrm{~cm}^{-1}\left(\mathrm{CH}_{3} \delta_{\text {as }}\right)$, whereas a loss of aliphatic methylene character was observed at $2958 \mathrm{~cm}^{-1}\left(\mathrm{CH}_{2} \nu_{\mathrm{as}}\right)$, $2847 \mathrm{~cm}^{-1}\left(\mathrm{CH}_{2} \nu_{\mathrm{s}}\right)$, and $1450 \mathrm{~cm}^{-1}\left(\mathrm{CH}_{2} \delta\right.$ scissor $)$. The largest change in aliphatic groups occurred with a negative peak at $2926 \mathrm{~cm}^{-1}$ and was assigned as a methylene stretching band. A variety of $\mathrm{C}-\mathrm{F}_{2}$ stretching vibrations were present from 1300 to $800 \mathrm{~cm}^{-1}$, all positive in the subtraction spectra. Large positive peaks associated with the PVDF backbone were assigned to the $\mathrm{CH}_{2}$ scissor mode $\left(1400 \mathrm{~cm}^{-1}\right), \mathrm{CF}_{2} / \mathrm{CH}_{2}$ twisting $\left(882 \mathrm{~cm}^{-1}\right)$, and $\mathrm{CF}_{2} / \mathrm{CH}_{2}$ skeletal $\beta$ twisting (840 $\left.\mathrm{cm}^{-1}\right)$. The increase in the PVDF vibrational modes indicates that the component exists in greater proportions at the surface of the coating. In fact, after $4000 \mathrm{~h}$, the ATR-FTIR spectrum indicated that the surfaces of the films without nanoclays were nearly devoid of any acrylic character in the polymer and were nearly purely PVDF. The smallest difference peaks were observed for the perfluoroalkyl clay composites using ATRFTIR spectroscopy. It has been hypothesized that smectites can act as free radical quenchers during UV exposure, ${ }^{44}$ which could also explain the reduction in photodegradation observed when the nanoclays were incorporated.

Surface ablation of the coatings was confirmed by thin film $\mathrm{X}$-ray diffraction (TF-XRD) (data shown in Figure S5), where the spectra show two important features: (1) a loss of film thickness and (2) surface enrichment of crystalline PVDF. The film that thinned most and showed the greatest increase in PVDF crystallinity after weathering was the polymer-only film. TF-XRD data also showed that addition of nanoclays reduced the film loss and changes in polymer crystallinity. Material loss can also be monitored by relative changes in the film thickness and gloss measurements, data for which are listed in Table 2 for

Table 2. Gloss and Thickness of the Films before and after $4000 \mathrm{~h}$ QUV-B

\begin{tabular}{lccc} 
& $\begin{array}{c}\text { gloss }(\% R) \\
\text { initial }\end{array}$ & $\begin{array}{c}\text { gloss }(\% \Delta R) \\
\text { QUV } 4 \mathrm{k} \mathrm{h}\end{array}$ & $\begin{array}{c}\text { thickness }(\% \Delta D) \\
\text { QUV } 4 \mathrm{k} \mathrm{h}\end{array}$ \\
PVDF & $12.6 \pm 1.1$ & $-33.3 \pm 2.9$ & $-36.5 \pm 9.8$ \\
PVDF + LNa & $12.6 \pm 0.3$ & $-35.7 \pm 0.96$ & $-29.4 \pm 8.5$ \\
PVDF + LAPc & $13.2 \pm 0.4$ & $-37.9 \pm 1.2$ & $-43.3 \pm 7.5$ \\
PVDF + LFPc & $9.1 \pm 0.2$ & $-13.2 \pm 0.44$ & $-23.1 \pm 2.1$ \\
\hline
\end{tabular}

each coating after weathering by QUV. Contrary to what was predicted, addition of the LNa clay did not appreciably affect gloss of the PVDF coating before or after weathering but slightly prevented the film loss over time. LFPc films had the lowest initial gloss compared with all of the other films (having a more matte appearance is often considered to be an aesthetic improvement for coatings, to reduce glare). LFPc films also showed the least change in gloss and the smallest change in the film thickness $(<30 \%)$ after weathering. Because the chemical functionality of the APTMS silane is more similar to that of the ester pendant groups in the acrylic resin, modified LAPc clays 


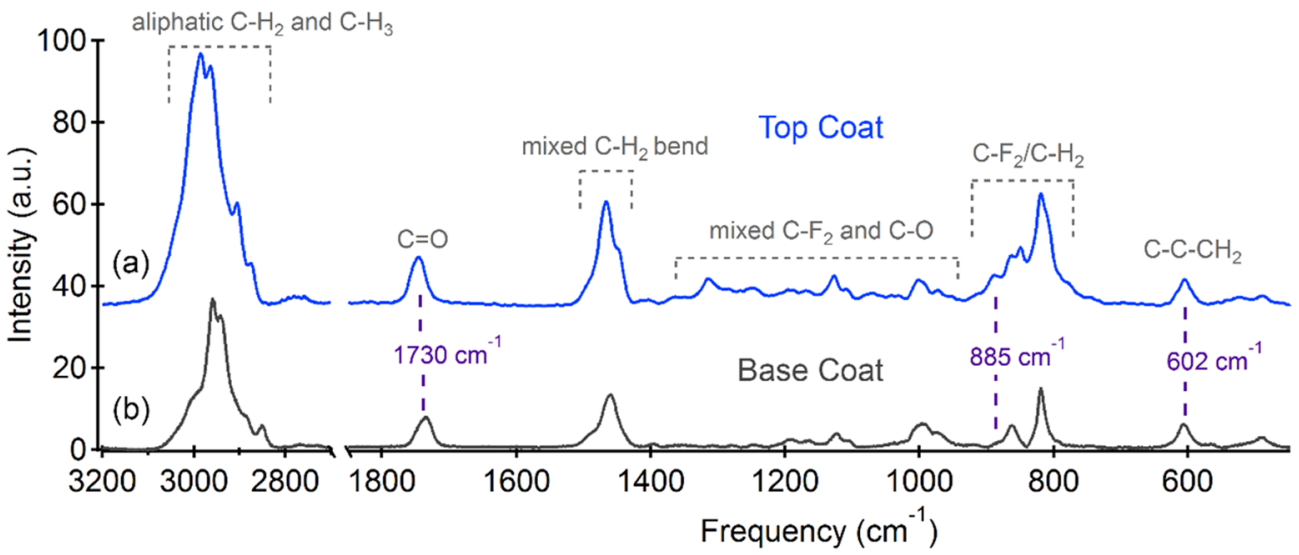

(c)

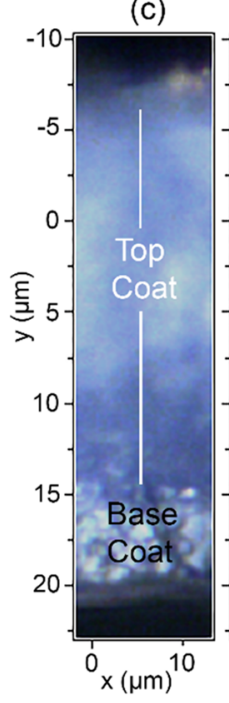

(d)

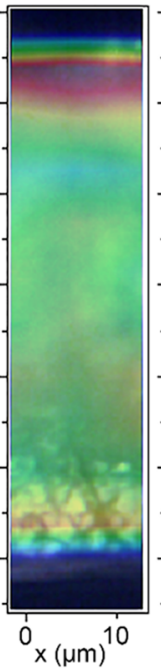

(e)



(f)

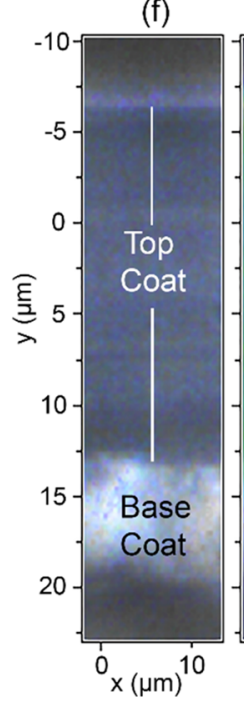

(g)

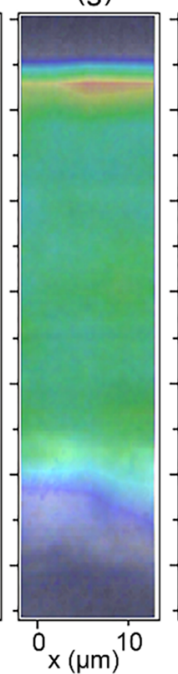

(h)

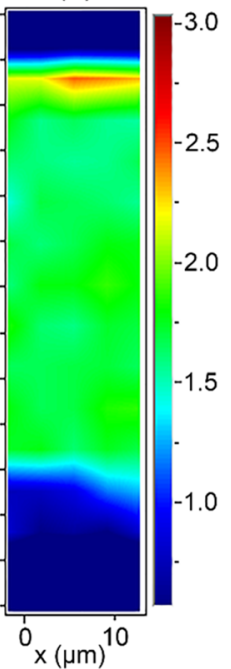

Figure 7. Raman spectra before weathering of the PVDF/acrylic top coat (a) and acrylic PMMA/PEA primer coat (b) normalized to the band at 602 $\mathrm{cm}^{-1}(\delta \mathrm{C}-\mathrm{C})$. Cross sections of weathered PVDF $(\mathrm{c})$ and PVDF $+\mathrm{LNa}(\mathrm{f})$ were mapped by Raman spectroscopy, and the intensity ratios of key PVDF/acrylic stretching bands are plotted $\left(I_{885} / I_{1730}\right)$ in $(\mathrm{e})$ and $(\mathrm{h})$. For comparison, the Raman maps were superimposed over white light images in (d) and (g). After normalization, the maximum intensity ratio was 3.0 and the minimum was 0.5 . Measured intensities were smoothed in both $x$ and $y$ directions to create a cohesive map; the blue fringes at the top and bottom are artifacts of smoothing to zero.

may be more susceptible to chemical degradation than clays of LFPc. If LAPc loses chemical functionality, the clay could be washed out from the coating matrix, resulting in a loss of the film due to the loss of radical quenchers in the clay.

Structural changes in the polymer that result in an increase in the crystallinity should give rise to a film that has excellent barrier properties. It has been observed in the literature that irradiation is capable of inducing conformational changes in semicrystalline fluoropolymers. ${ }^{45,46}$ On the basis of our ATRFTIR data, the percentage of PVDF at the surface increased to near purity during weathering, and on the basis of TF-XRD data, the films became more crystalline. The EIS stability of particularly the LFPc film during weathering suggests that the films weather in two phases: (1) microcrystalline PVDFenriched domains and (2) nanocrystalline-modified clays, with both phases having electrolyte/corrosive impenetrable crystalline domains. It is also likely that the nanoclays might (1) as suggested by others ${ }^{44}$ both absorb and scatter incoming UV radiation, thus minimizing the amount penetrating the coating, and (2) reduce photodegradation-induced mass transport out of the film. Raman microscopy was used to investigate these claims and produce cross-section maps of a polymer-only film and a polymer + LNa film after weathering, both of which are shown in Figure 7. The polymer-only film showed a higher intensity (concentration) and a thicker enriched layer of PVDF in the film (or, conversely, the acrylic-depleted layer was thicker) compared with the nanocomposite film after weathering for $4500 \mathrm{~h}$. The acrylic base coat was significantly degraded and embrittled when a polymer-only top coat was used as can be observed by the cracks in the lower portion of the cross section in Figure $7 c$ and by the accompanying heat map of the ratio of key PVDF/acrylic Raman peaks.

\section{CONCLUSIONS}

We have demonstrated that by incorporating chemically modified nanoclays, the barrier properties of films produced from aqueous dispersions of acrylic/PVDF lattices can be significantly improved over both the short and long term. XPS and Raman data showed that (1) Laponite clay was covalently modified with FOTES and cation exchanged with Pc; (2) grafting efficiency was more than $100 \%$, suggesting that each accessible hydroxyl along the clay rim was covalently bound to one FOTES molecule; (3) approximately more than 50\% silanes remaining after washing were covalently cross-linked to other FOTES molecules; and (4) cation exchange with Pc increased the hydrophilicity of the covalently modified clay. 
Covalently modified nanoclay particles were dispersible in aqueous colloidal suspensions of a highly weatherable resin and with a much smaller loading weight $(0.6 \%$ of a dry film) than other studies have reported. ${ }^{10,11,21}$

The weathering performance of the nanocomposite films was better in every measured parameter than in the films without the clay. All composite films studied retained their highperformance barrier properties, as measured by EIS and the stress index, after a substantial loss of acrylic functionality near the surface and a loss of $10-40 \%$ of the film thickness. However, the performance characteristics of each differed considerably. Although the PVDF/acrylic waterborne resin is an exceptional barrier top coat with long-term weatherability, its performance can be substantially improved by the inclusion of chemically compatible nanoclays.

What accounts for the better EIS barrier performance of LFPc compared with LAPc composite films during weathering? It seems likely that the interaction between the polymer resin and the organofunctionalized clays may play a substantial role. The latex polymer used in these studies was synthesized in a core-shell synthesis, with the core and the shell having both acrylic and PVDF components but in differing amounts. ${ }^{2,3}$ To enable dispersion in aqueous media, the shell has a greater percentage of acrylic (hydrophilic) than the core does. Given that the best barrier properties were observed in films with added LFPc, it is possible that the LFPc clays facilitate the formation of an interpenetrating polymer network better than those with LAPc.

Good stability (both chemical and mechanical) was observed by the low stress index of chemically modified clays in the PVDF/acrylic films. The LNa and LFPc clays had the highest degree of exfoliation and the longest time constants for much of the weathering time. However, the unmodified LNa composite film had a lower film resistance and higher coating capacitance than the LFPc composite film. One explanation for the LNa composite film's diminished barrier properties despite having a longer time constant may arise from the microstructural arrangement of the clays in the interstitial spaces of the wet resin during the film formation. Evidence suggests that although LNa was exfoliated completely in water, there was little chemical compatibility with the latex particles in the film, which can disrupt coalescence of latex particles during drying. The result would be larger voids between latex particles where migrating ion species are retained, as was indeed suggested by the mass-transport studies. Chemical modification of clays resulted in particles that retained their ability to be exfoliated and improved their dispersibility in this highly weatherable resin. The combination of the microstructural arrangement of clay particles, the chemical compatibility between the perfluoroalkyl-modified clays and the PVDF portion of the resin, and the excellent weatherability of both perfluoroalkyl and PVDF functionalities likely accounts for the superior corrosion resistance of the LFPc composite films throughout weathering.

\section{ASSOCIATED CONTENT}

\section{S Supporting Information}

The Supporting Information is available free of charge on the ACS Publications website at DOI: 10.1021/acsomega.6b00091.

Extended discussion of EIS and circuit element trends, TF-XRD of weathered nanocomposites, and surface characterization of functionalized nanoclays with Raman spectroscopy, WAXD, TGA, and XPS (PDF)

\section{AUTHOR INFORMATION}

\section{Corresponding Author}

*E-mail: claret@pdx.edu.

\section{Notes}

The authors declare no competing financial interest.

\section{ACKNOWLEDGMENTS}

We would like to thank Anne Bentley, Lewis \& Clark College, for the use of the zeta potentiometer and Andrea Goforth, Portland State University, for the use of the X-ray diffractometer. This project received funding support from the National Science Foundation (CHE-1139230, MRI DMR0923572, and MRI DMR-122963) and Portland State University.

\section{REFERENCES}

(1) Clare, T. L.; Lins, P. A. Evaluation of fluorinated protective coatings for outdoor metals; In Proceedings of Metal 07: Interim Meeting of the ICOM-CC Metal WG; Degrigny, C., Lang, R. V., Joosten, I., Ankersmith, B., Eds.; ICOM: Rijksmuseum, Amsterdam, 2007; pp 8387.

(2) Iezzi, R. A.; Gaboury, S.; Wood, K. Acrylic-fluoropolymer mixtures and their use in coatings. Prog. Org. Coat. 2000, 40, 55-60.

(3) Wood, K. A. How can we effectively use accelerated methods to predict the decorative properties of PVDF-based coatings? - A practical approach. Prog. Org. Coat. 2014, 77, 2140-2146.

(4) Zhou, H.; Wang, H.; Niu, H.; Gestos, A.; Lin, T. Robust, SelfHealing Superamphiphobic Fabrics Prepared by Two-Step Coating of Fluoro-Containing Polymer, Fluoroalkyl Silane, and Modified Silica Nanoparticles. Adv. Funct. Mater. 2013, 23, 1664-1670.

(5) Swartz, N. A.; Wood, K. A.; Clare, T. L. Characterizing and improving performance properties of thin solid films produced by weatherable water-borne colloidal suspensions on bronze substrates. Prog. Org. Coat. 2012, 75, 215-223.

(6) Kovacs, J. R.; Liu, C.; Hammond, P. T. Spray Layer-by-Layer Assembled Clay Composite Thin Films as Selective Layers in Reverse Osmosis Membranes. ACS Appl. Mater. Interfaces 2015, 7, 1337513383.

(7) Xiang, F.; Tzeng, P.; Sawyer, J. S.; Regev, O.; Grunlan, J. C. Improving the Gas Barrier Property of Clay-Polymer Multilayer Thin Films Using Shorter Deposition Times. ACS Appl. Mater. Interfaces 2014, 6, 6040-6048.

(8) Tokudome, Y.; Hara, T.; Abe, R.; Takahashi, M. Transparent and Robust Siloxane-Based Hybrid Lamella Film As a Water Vapor Barrier Coating. ACS Appl. Mater. Interfaces 2014, 6, 19355-19359.

(9) Zengeni, E.; Hartmann, P. C.; Pasch, H. Encapsulation of Clay by Ad-Miniemulsion Polymerization: The Influence of Clay Size and Modifier Reactivity on Latex Morphology and Physical Properties. ACS Appl. Mater. Interfaces 2012, 4, 6957-6968.

(10) Negrete-Herrera, N.; Putaux, J.-L.; David, L.; De Haas, F.; Bourgeat-Lami, E. Polymer/Laponite composite latexes: particle morphology, film microstructure, and properties. Macromol. Rapid Commun. 2007, 28, 1567-1573.

(11) Wheeler, P. A.; Wang, J.; Mathias, L. J. Poly(methyl methacrylate)/laponite nanocomposites: Exploring covalent and ionic clay modifications. Chem. Mater. 2006, 18, 3937-3945.

(12) Das, P.; Schipmann, S.; Malho, J.-M.; Zhu, B.; Klemradt, U.; Walther, A. Facile Access to Large-Scale, Self-Assembled, NacreInspired, High-Performance Materials with Tunable Nanoscale Periodicities. ACS Appl. Mater. Interfaces 2013, 5, 3738-3747.

(13) Qian, Y.; Lindsay, C. I.; Macosko, C.; Stein, A. Synthesis and Properties of Vermiculite-Reinforced Polyurethane Nanocomposites. ACS Appl. Mater. Interfaces 2011, 3, 3709-3717. 
(14) Talbot, E. L.; Yang, L.; Berson, A.; Bain, C. D. Control of the Particle Distribution in Inkjet Printing through an Evaporation-Driven Sol-Gel Transition. ACS Appl. Mater. Interfaces 2014, 6, 9572-9583.

(15) Ruzicka, B.; Zaccarelli, E. A fresh look at the Laponite phase diagram. Soft Matter 2011, 7, 1268-1286.

(16) Wilson, R.; Plivelic, T. S.; Ramya, P.; Ranganathaiah, C.; Kariduraganavar, M. Y.; Sivasankarapillai, A. K.; Thomas, S. Influence of Clay Content and Amount of Organic Modifiers on Morphology and Pervaporation Performance of EVA/Clay Nanocomposites. Ind. Eng. Chem. Res. 2011, 50, 3986-3993.

(17) Tawari, S. L.; Koch, D. L.; Cohen, C. Electrical double-layer effects on the Brownian diffusivity and aggregation rate of Laponite clay particles. J. Colloid Interface Sci. 2001, 240, 54-66.

(18) Herrera, N. N.; Letoffe, J.-M.; Reymond, J.-P.; Bourgeat-Lami, E. Silylation of Laponite clay particles with monofunctional and trifunctional vinyl alkoxysilanes. J. Mater. Chem. 2005, 15, 863-871.

(19) Herrera, N. N.; Letoffe, J.-M.; Putaux, J.-L.; David, L.; BourgeatLami, E. Aqueous Dispersions of Silane-Functionalized Laponite Clay Platelets. A First Step toward the Elaboration of Water-Based Polymer/Clay Nanocomposites. Langmuir 2004, 20, 1564-1571.

(20) Herrera, N. N.; Putaux, J.-L.; Bourgeat-Lami, E. Synthesis of polymer/Laponite nanocomposite latex particles via emulsion polymerization using silylated and cation-exchanged Laponite clay platelets. Prog. Solid State Chem. 2006, 34, 121-137.

(21) Negrete-Herrera, N.; Putaux, J.-L.; David, L.; Bourgeat-Lami, E. Polymer/Laponite Composite Colloids through Emulsion Polymerization: Influence of the Clay Modification Level on Particle Morphology. Macromolecules 2006, 39, 9177-9184.

(22) Macdonald, D. D. Reflections on the history of electrochemical impedance spectroscopy. Electrochim. Acta 2006, 51, 1376-1388.

(23) van Westing, E. P. M.; Ferrari, G. M.; de Wit, J. H. W. The Determination of Coating Performance with Impedance Measurements II. Water-Uptake of Coatings. Corros. Sci. 1994, 36, 957-977.

(24) Swartz, N. A.; Clare, T. L. Understanding the differences in film formation mechanisms of two comparable solvent based and waterborne coatings on bronze substrates by electrochemical impedance spectroscopy. Electrochim. Acta 2012, 62, 199-206.

(25) Ellingson, L. A.; Shedlosky, T. J.; Bierwagen, G. P.; de la Rie, E. R.; Brostoff, L. B. The Use of Electrochemical Impedance Spectroscopy in the Evaluation of Coatings for Outdoor Bronze. Stud. Conserv. 2004, 49, 53-62.

(26) Perrotta, A.; García, S. J.; Michels, J. J.; Andringa, A.-M.; Creatore, M. Analysis of Nanoporosity in Moisture Permeation Barrier Layers by Electrochemical Impedance Spectroscopy. ACS Appl. Mater. Interfaces 2015, 7, 15968-15977.

(27) Castela, A. S.; Simões, A. M. An impedance model for the estimation of water absorption in organic coatings. Part I: A linear dielectric mixture equation. Corros. Sci. 2003, 45, 1631-1646.

(28) Liu, B.; Li, Y.; Lin, H.; Cao, C.-N. Electrochemical impedance spectroscopy study on the diffusion behavior of water through epoxy coatings. Corrosion 2003, 59, 817-820.

(29) Hu, J.-M.; Zhang, J.-T.; Zhang, J.-Q.; Cao, C.-N. A novel method for determination of diffusion coefficient of corrosive species in organic coatings by EIS. J. Mater. Sci. 2004, 39, 4475-4479.

(30) Philippe, L. V. S.; Lyon, S. B.; Sammon, C.; Yarwood, J. Validation of electrochemical impedance measurements for water sorption into epoxy coatings using gravimetry and infra-red spectroscopy. Corros. Sci. 2008, 50, 887-896.

(31) Lazzari, M.; Chiantore, O. Thermal-ageing of paraloid acrylic protective polymers. Polymer 2000, 41, 6447-6455.

(32) Ćurković, H. O.; Kosec, T.; Marušić, K.; Legat, A. An electrochemical impedance study of the corrosion protection of artificially formed patinas on recent bronze. Electrochim. Acta 2012, 83, 28-39.

(33) Cano, E.; Lafuente, D.; Bastidas, D. M. Use of EIS for the evaluation of the protective properties of coatings for metallic cultural heritage: a review. J. Solid State Electrochem. 2010, 14, 381-391.
(34) Shashoua, Y.; Matthiesen, H. Protection of iron and steel in large outdoor industrial heritage objects. Corros. Eng. Sci. Technol. 2010, 45, 357-361.

(35) Clare, T. L.; Swartz, N. A. Characterization of high performance protective coatings for use on culturally significant works; In Intelligent Coatings for Corrosion Control; Tiwari, A., Hihara, L., Rawlins, J., Eds.; Butterworth-Heinemann, 2014; pp 641-671.

(36) Taddei, P.; Balducci, F.; Simoni, R.; Monti, P. Raman, IR and thermal study of a new highly biocompatible phosphorylcholine-based contact lens. J. Mol. Struct. 2005, 744-747, 507-514.

(37) Cousin, F.; Cabuil, V.; Grillo, I.; Levitz, P. Competition between Entropy and Electrostatic Interactions in a Binary Colloidal Mixture of Spheres and Platelets. Langmuir 2008, 24, 11422-11430.

(38) Lewin, M. Reflections on migration of clay and structural changes in nanocomposites. Polym. Adv. Technol. 2006, 17, 758-763.

(39) Hinderliter, B. R.; Croll, S. G. Simulation of transient electrochemical impedance spectroscopy due to water uptake or oxide growth. Electrochim. Acta 2009, 54, 5344-5352.

(40) Hinderliter, B. R.; Croll, S. G.; Tallman, D. E.; Su, Q.; Bierwagen, G. P. Interpretation of EIS data from accelerated exposure of coated metals based on modeling of coating physical properties. Electrochim. Acta 2006, 51, 4505-4515.

(41) Jung, H.; Kim, H.-M.; Choy, Y. B.; Hwang, S.-J.; Choy, J.-H. Laponite-based nanohybrid for enhanced solubility and controlled release of itraconazole. Int. J. Pharm. 2008, 349, 283-290.

(42) Otieno-Alego, V.; Heath, G.; Hallam, D.; Creagh, D. Electrochemical evaluation of the anti-corrosion performance of waxy coatings for outdoor bronze conservation; In Conference internationale sur la conservation des métaux; James \& James: London, 1998; pp 309-314.

(43) Bedoya, F. E.; Gallego, L. M.; Bermúdez, A.; Castaño, J. G.; Echeverría, F.; Calderón, J. A.; Maya, J. G. New strategy to assess the performance of organic coatings during ultraviolet condensation weathering tests. Electrochim. Acta 2014, 124, 119-127.

(44) Essawy, H. A.; Abd El-Wahab, N. A.; Abd El-Ghaffar, M. A. PVC-Laponite nanocomposites: Enhanced resistance to UV radiation. Polym. Degrad. Stab. 2008, 93, 1472-1478.

(45) Gu, X.; Michaels, C. A.; Nguyen, D.; Jean, Y. C.; Martin, J. W.; Nguyen, T. Surface and interfacial properties of PVDF/acrylic copolymer blends before and after UV exposure. Appl. Surf. Sci. 2006, 252, 5168-5181.

(46) Horibe, H.; Hosokawa, Y.; Oshiro, H.; Sasaki, Y.; Takahashi, S.; Kono, A.; Nishiyama, T.; Danno, T. Effect of heat-treatment temperature after polymer melt and blending ratio on the crystalline structure of PVDF in a PVDF/PMMA blend. Polym. J. 2013, 45, $1195-1201$. 